\title{
The role of hyperthermic intraperitoneal chemotherapy in the treatment of spontaneously ruptured hepatocellular carcinoma: a pilot study
}

\author{
Shiye Ruan ${ }^{1,2 \#}$, Ning Shi ${ }^{1 \#}$, Zhihong Chen ${ }^{1}$, Hongwei Han ${ }^{1,2}$, Hanyue Wang ${ }^{3}$, Liang Jin ${ }^{1}$, Yiping Zou ${ }^{1}$, \\ Yuanpeng Zhang ${ }^{1}$, Min Yu ${ }^{1}$, Haosheng Jin ${ }^{1,2}$ \\ ${ }^{1}$ Department of General Surgery, Guangdong Provincial People's Hospital, Guangdong Academy of Medical Sciences, Guangzhou, China; ${ }^{2}$ The \\ Second School of Clinical Medicine, Southern Medical University, Guangzhou, China; ${ }^{3}$ Department of Pathology, Jinan University, Guangzhou, \\ China \\ Contributions: (I) Conception and design: H Jin, M Yu, S Ruan, N Shi; (II) Administrative support: H Jin, M Yu; (III) Provision of study materials or \\ patients: None; (IV) Collection and assembly of data: S Ruan, Z Chen, H Wang, H Han, Y Zou, Y Zhang; (V) Data analysis and interpretation: S \\ Ruan, Z Chen, H Wang, H Han, Y Zou, Y Zhang; (VI) Manuscript writing: All authors; (VII) Final approval of manuscript: All authors. \\ \#These authors contributed equally to this work. \\ Correspondence to: Min Yu; Haosheng Jin. Department of General Surgery, Guangdong Provincial People's Hospital, Guangdong Academy of Medical \\ Sciences, Guangzhou, China. Email: yumin@gdph.org.cn; thundercry@163.com.
}

Background: Spontaneous tumor rupture is a distinctive disease pattern in patients with hepatocellular carcinoma (HCC). The application of hyperthermic intraperitoneal chemotherapy (HIPEC) in spontaneously ruptured hepatocellular carcinoma (srHCC) is debatable. Our study aimed to compare the long-term outcomes of srHCC $v s$. nrHCC and to test the role of postoperative HIPEC in patients with srHCC after hepatectomy.

Methods: From 2014 to 2018, PSM was performed to compare 57 patients who performed liver resection for srHCC and met the research criteria with 57 nrHCC patients selected from 446 consecutive patients. Then patients with srHCC were divided into two groups according to whether they underwent HIPEC after hepatectomy.

Results: After 1:1 PSM, the clinical characteristics of the patients with srHCC and nrHCC were comparable. In terms of long-term outcomes, the nrHCC group had significantly longer OS $(\mathrm{P}=0.026)$ and DFS $(\mathrm{P}<0.001)$ than the srHCC group. Of the 57 srHCC patients, the HIPEC group showed added complications compared to the non-HIPEC group, including an increased length of hospital stay and higher in-hospital costs. However, there were no significant differences in the metastatic patterns of these recurrent patients, and there was no statistically significant difference in DFS $(\mathrm{P}=0.28)$ or OS $(\mathrm{P}=0.56)$ between the two groups.

Conclusions: The prognosis of ruptured HCC patients were worse than those of non-ruptured HCC patients. HIPEC may not be a robust treatment for srHCC now.

Keywords: Hyperthermic intraperitoneal chemotherapy (HIPEC); spontaneous rupture; hepatocellular carcinoma (HCC); propensity score matching

Submitted Jun 05, 2020. Accepted for publication Sep 08, 2020.

doi: 10.21037/atm-20-5829

View this article at: http://dx.doi.org/10.21037/atm-20-5829 


\section{Introduction}

Liver cancer, ranking as the second-highest cause of cancer-related death, is one of the most common human malignancies around the world (1). In the last decades, advances in surgical techniques have made it possible for hepatocellular carcinoma (HCC) patients to live longer than in the past. However, the prognosis is still poor in patients who suffer from spontaneously ruptured HCC (srHCC) (2). Peritoneal and hepatic implantation metastasis, considered caused by exfoliated HCC cells, is the most often observed pattern of recurrence or metastasis in these patients (3). The more effective method to remove free HCC cells in the abdominal cavity deserves further exploration.

Peritoneal lavage with distilled water (DWPL), extensively applied for clearing bacteria and free tumor cells in the peritoneal cavity is a conventional technique developed to reduce the incidence of metastases. However, cancer cells can survive in a hypotonic condition, and the efficacy of this therapy is further affected by the contamination of the water in vivo and the "inoculum size" of exfoliated cancer cells (3-5).

Intraperitoneal chemotherapy can be conducted at an elevated temperature, defined as hyperthermic intraperitoneal chemotherapy (HIPEC). Hyperthermia can increase the permeability of chemotherapy on the peritoneal surface and enhance the sensitivity of the tumor to chemotherapy by interfering with DNA repair. It also induces apoptosis and activates heat-shock proteins that serve as receptors for natural killer cells, inhibits angiogenesis, and has a direct cytotoxic effect by promoting the denaturation of proteins (6-9). With women with advanced ovarian cancer, HIPEC resulted in more prolonged survival and could significantly improve their prognoses (10). Also, some randomized controlled trials (RCTs) support using this treatment in colorectal cancer $(11,12)$. Moreover, some recent study suggested that cytoreductive surgery followed by HIPEC gives the patient a chance for a good relapse free and overall survival and might be considered as an option in highly patients (13-15). On this basis, we hypothesized HIPEC could improve the long-term outcomes of srHCC. Recent research from Chen suggested that fluorouracil implants can mitigate the risk of peritoneal and hepatic dissemination after HCC rupture (16). Therefore, our research aimed to estimate the safety and efficacy of using HIPEC in patients to treat srHCC. We present the following article in accordance with the STROBE reporting checklist. (available at http://dx.doi. org/10.21037/atm-20-5829).

\section{Methods}

\section{Patients}

Data collection was retrospective. Seven hundred seventyfour patients were found from a prospectively maintained database at the Guangdong Provincial People's Hospital in China between 2014 and 2018. A multidisciplinary team first assessed all patients. Then we operated on patients with excellent liver function. The inclusion criteria were Child's A or better liver disease and no clinical evidence of significant portal hypertension. After excluding patients according to the pre-specified criteria, among the remaining 503 patients, $446 \mathrm{nrHCC}$ patients and 57 srHCC patients were eventually included for further analysis (Figure 1). The study was conducted in accordance with the Declaration of Helsinki (as revised in 2013). Institutional review board approval of our hospital was obtained for this study. This was a retrospective clinical study, which only analyzed the earlier clinical data. The data processed did not reveal the patient's identity information, so there was no need for ethical recognition and informed consent.

\section{Procedure and HIPEC outcome measure}

Then, the patients with srHCC were divided into two groups according to whether they underwent HIPEC after hepatectomy. In the HIPEC group, the silicon perfusion catheters were placed at the end of the laparoscopic hepatectomy or open radical surgery. HIPEC was administered 2 times on the $3 \mathrm{rd}$ and 5 th days after the operation in 30 patients (17-23). The detailed information concerning the HIPEC process can be found in the protocol, uploaded as a supplementary file. The temperature in the abdominal cavity was supported at $43{ }^{\circ} \mathrm{C}\left(109^{\circ} \mathrm{F}\right)$ by circulating the heated saline. Perfusion with 5 -fluorouracil $\left(1,000 \mathrm{mg} / \mathrm{m}^{2}\right)(24,25)$ or lobaplatin $\left(50 \mathrm{mg} / \mathrm{m}^{2}\right)(26-30)$ was then started at a flow rate of 300 to 600 milliliters per minute to cause the entire abdomen to be filled with the perfusate. The treatment supported 90 minutes holding the 60-minute perfusion period. At the end of the procedure, perfusion catheters were used to drain the perfusion as thoroughly as possible.

\section{Follow-up}

Follow-up data were obtained by outpatient service and telephone consultation for patients and reviewing the medical record from the database. The patients 


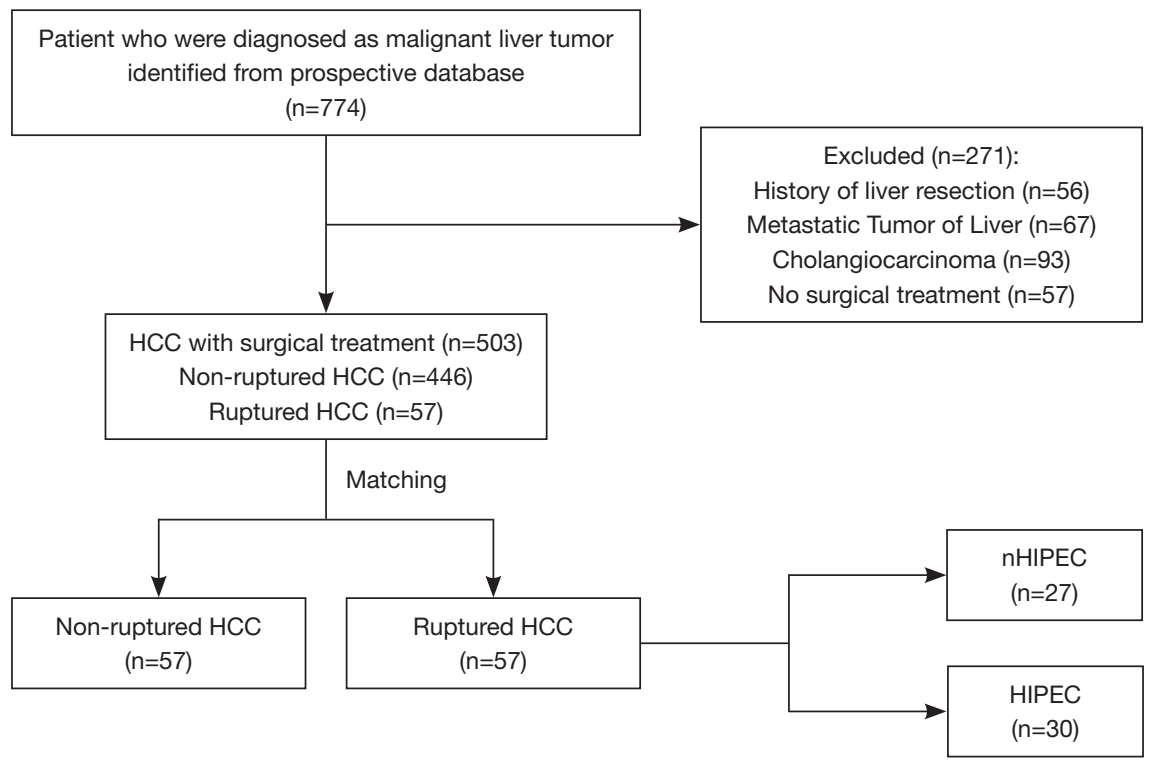

Figure 1 Flow chart showing patient enrollment and surgical treatment strategies. HCC, hepatocellular carcinoma; HIPEC, hyperthermic intraperitoneal chemotherapy.

were followed up every 3 months in the first year after hepatectomy and every 3 to 6 months after that. The recurrence and metastasis of patients were estimated by analyzing their AFP levels and the results of ultrasonography, contrast-enhanced CT, or MRI. Overall survival (OS) is defined as the interval between the surgery date and death for any reason. Disease-free survival (DFS) time was regarded as the time after surgery, during which the patient survived with no evidence of HCC. Patients who had no documented evidence of events were censored at the date of the last follow-up. The censoring date of the present study was May 30, 2019.

Morbidity was defined as any complication observed during hospitalization or within 30 days after the procedure. On the Clavien-Dindo classification, the details of postoperative complications are categorized (31). The following postoperative outcomes were tested: postoperative bleeding, bile leakage, hepatic dysfunction, pulmonary complications, reoperation, and mortality within 30 days of the surgery. The definition of the postoperative complications is according to current evidence (32-34).

\section{Propensity score matching}

Propensity-score matching is a statistical method that can find a group of cases with similar baseline features. The propensity score, estimated using a non-parsimonious multivariable logistic-regression model, is a conditional probability of having a particular exposure (HCC with ruptured versus non-ruptured HCC) given a set of measured baseline measured covariates (35-37). We performed matching by a 1:1 matching protocol without replacement, with a caliper width equal to 0.2. Equilibrium is assessed for all the baseline variables to estimate the prematch imbalance and postmatch balance. A $\mathrm{P}$ value that is greater than 0.05 for a variable after matching shows a slight imbalance.

\section{Statistical analysis}

Paired comparisons were conducted by using McNemar's tests for binary variables expressed as the number and percentage of subjects, and a paired Student's $t$-tests or paired-sample tests were performed for continuous variables described as the mean values and standard deviation. The Kaplan-Meier survival analysis was conducted to estimate differences in patient OS and DFS between the two groups. Cox proportional hazard regression analysis was used to find independent prognostic factors. R 3.6.1 software conducted all statistical analyses for Windows. 
Table 1 Characteristics before and after propensity score matching

\begin{tabular}{|c|c|c|c|c|c|c|}
\hline Variables & \multicolumn{3}{|c|}{ Before matching } & \multicolumn{3}{|c|}{ After matching } \\
\hline Male & 48 (84.2) & $392(87.9)$ & 0.429 & $48(84.2)$ & $43(75.4)$ & 0.243 \\
\hline Age $($ mean $\pm S D)$ & $50.7 \pm 13.9$ & $53.9 \pm 11.5$ & 0.019 & $50.7 \pm 13.9$ & $53.3 \pm 13.0$ & 0.301 \\
\hline Solitary nodule & $50(87.7)$ & $360(80.7)$ & 0.199 & $50(87.7)$ & $56(98.2)$ & 0.061 \\
\hline LLR & $22(38.6)$ & $194(43.5)$ & 0.456 & $22(38.6)$ & $24(42.1)$ & 0.698 \\
\hline Serum ALT (mean \pm SD) & $45.6 \pm 40.7$ & $50.6 \pm 54.1$ & 0.403 & $45.6 \pm 40.7$ & $49.0 \pm 45.2$ & 0.666 \\
\hline ALB $($ mean $\pm \mathrm{SD})$ & $36.4 \pm 5.7$ & $38.3 \pm 17.3$ & 0.819 & $36.4 \pm 5.7$ & $37.7 \pm 5.5$ & 0.189 \\
\hline PVTT & $5(8.8)$ & $19(4.3)$ & 0.132 & $52(91.2)$ & $54(94.7)$ & 0.716 \\
\hline MVI & $23(40.1)$ & $134(30.0)$ & 0.122 & $23(40.4)$ & $21(36.8)$ & 0.700 \\
\hline Tumor size $($ mean $\pm \mathrm{SD})$ & $8.1 \pm 4.4$ & $5.4 \pm 3.6$ & 0.025 & $8.1 \pm 4.4$ & $7.1 \pm 4.4$ & 0.242 \\
\hline
\end{tabular}

All demographic and pathological variables with $\mathrm{P}<0.2$ are included in the logistic model. srHCC, spontaneously ruptured hepatocellular carcinoma; CAHB, chronic active hepatitis B; AFP, alpha-fetoprotein; LLR, laparoscopic liver resection; PVTT, portal vein, tumor thrombus; $\mathrm{MVI}$, microvascular invasion; PT, prothrombin time; SD, standard deviation.

\section{Results}

Propensity-score matching analysis to compare srHCC versus non-ruptured HCC groups

We obtained a 1:1 paired cohort (57 patients in each group) for the srHCC versus nrHCC comparison. The patient demographics and clinical characteristics before and after PSM are presented in Table 1. These groups were wellmatched for crucial confounders-i.e., age, chronic activated hepatitis B (CAHB), solitary nodule, portal vein, tumor thrombus (PVTT), microvascular invasion, and tumor size. After matching, there are still slight differences for some variables not contained in the PSM analysis (Table 1). However, the balance test revealed that the selected patients in the two cohorts were matched well. Figure 2 shows the density curve of the propensity scores in both groups before and after matching.

The 1-, 2- and 3-year DFS rates in the nrHCC patient were $66.0 \%, 63.5 \%$ and $55.6 \%$, respectively, and were $33.5 \%, 31.0 \%$, and $18.6 \%$, respectively, in the srHCC patient. The 1-, 2- and 3-year OS rates in the nrHCC group were $84.4 \%, 76.7 \%$ and $76.7 \%$, respectively, and were $72.7 \%, 58.2 \%$ and $38.3 \%$, respectively, in the srHCC group. The outcomes of OS and DFS were significantly better for patients without spontaneous rupture than for those with rupture after liver resection $(\mathrm{P}=0.029$ and $\mathrm{P}<0.001$, respectively; Figure 2).

\section{Hepatectomy versus hepatectomy combined with HIPEC for patients with srHCC}

\section{Clinicopathological characteristics}

The clinicopathological features and demographic and baseline disease characteristics of 57 patients for the two groups are shown in Table 2, and no statistical differences were attained between the HIPEC and non-HIPEC patients. No death occurred during the perioperative period. The mean operative time was $285.33 \pm 104.03$ minutes in the HIPEC group and $280.37 \pm 98.30$ minutes in the surgery only group, and $52.63 \%$ of patients required perioperative blood transfusion. The median total duration of hospitalization was $11.26 \pm 3.6$ days in the non-HIPEC 
A

Before matching

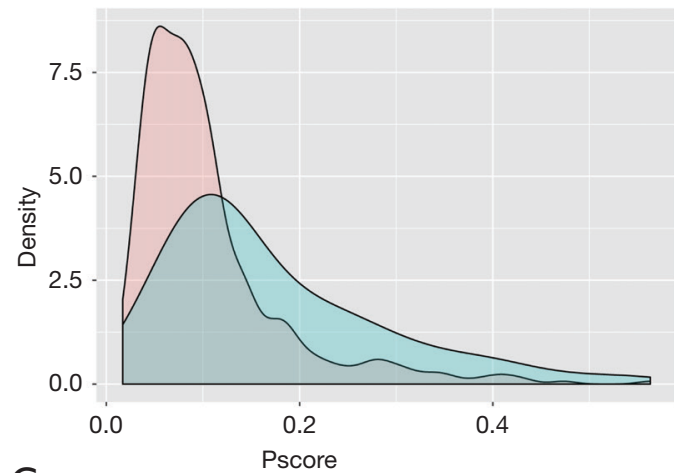

C

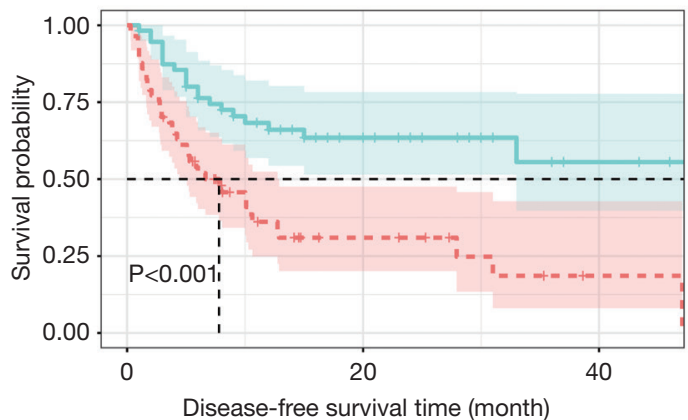

B
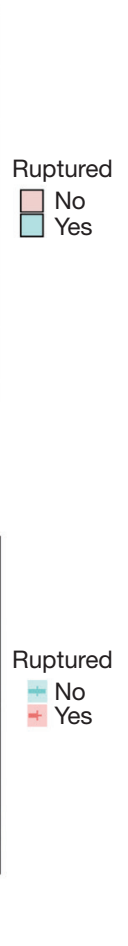
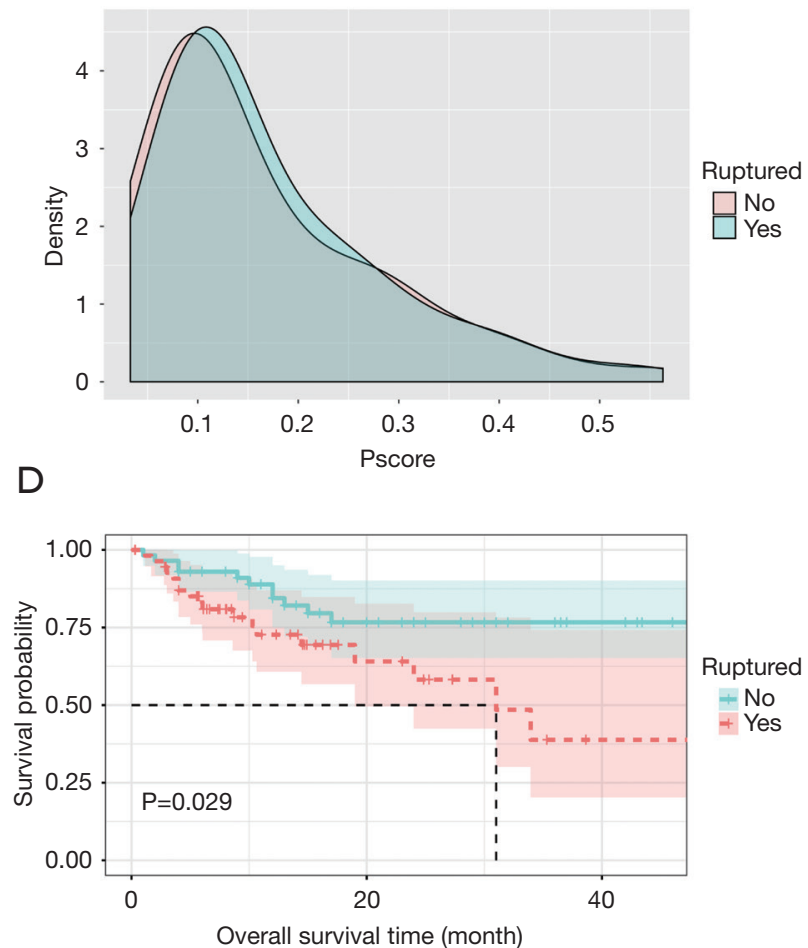

Figure 2 The overall balance test showed that the selected patients in the two groups matched well (A,B). Comparison of survival rates between the ruptured and non-ruptured groups. (C,D) Cumulative DFS and OS, measured before propensity matching and after propensity matching. OS, overall survival; DFS, disease-free survival.

group and $16.42 \pm 7.8$ days in the HIPEC group.

Clavien-Dindo grade I-II complications occurred in 14 cases, and grade III-IV morbidity occurred in 1 case among the 30 patients in the HIPEC group. Among the 27 patients in the non-HIPEC group, grade I-II complications occurred in 12 cases; grade III-IV morbidity occurred in 2 cases. The incidence of postoperative bleeding, peritoneal infection, hepatic dysfunction, and pulmonary complications is similar in the two groups. Patients who undergo HIPEC had a higher incidence of other complications than those having no HIPEC $(50.0 \%$ versus $11.1 \% ; \mathrm{P}=0.002)$; the difference was statistical significance. No significant differences were observed in the incidence rate of morbidity between the HIPEC and non-HIPEC groups $(\mathrm{P}=0.889)$. The mean hospitalization expenses were higher in the HIPEC patient than in the non-HIPEC patient $(\$ 16,946.82$ versus $\$ 8,934.29$, respectively; $\mathrm{P}<0.001)$. There were no postoperative deaths or reoperations in both groups (Table 2).

\section{Long-term outcomes}

Of the 57 patients found in the study, 30 (53.4\%) underwent HIPEC, and 27 (46.6\%) underwent surgery alone. After a median follow-up of 9.43 months, 44 of the 57 patients (77.19\%) had experienced a disease recurrence, and 17 of the 57 patients $(29.82 \%)$ died. The median DFS was longer in patients that underwent HIPEC after liver resection than in patients that had operation alone (10.07 versus 6.00 months, $\mathrm{P}=0.28$, Figure 3), but no statistically significant difference was attained. The long-term outcomes were not significantly improved in the HIPEC group compared to the non-HIPEC group after hepatectomy of srHCC.

The postoperative 3-month, 6-month, and 12-month DFS rates were $69.0 \%, 53.33 \%$, and $46.67 \%$, respectively, in the HIPEC group and were $77.78 \%, 48.15 \%$, and $33.33 \%$, respectively, in the non-HIPEC patients. The DFS rate in the HIPEC patient was higher than that in the nonHIPEC patient. No significant difference was observed for DFS between the two treatment arms $(\mathrm{P}=0.56$; Figure 3$)$. 
Table 2 Baseline clinical characteristics data of patients with srHCC undergoing operation or operation-HIPEC

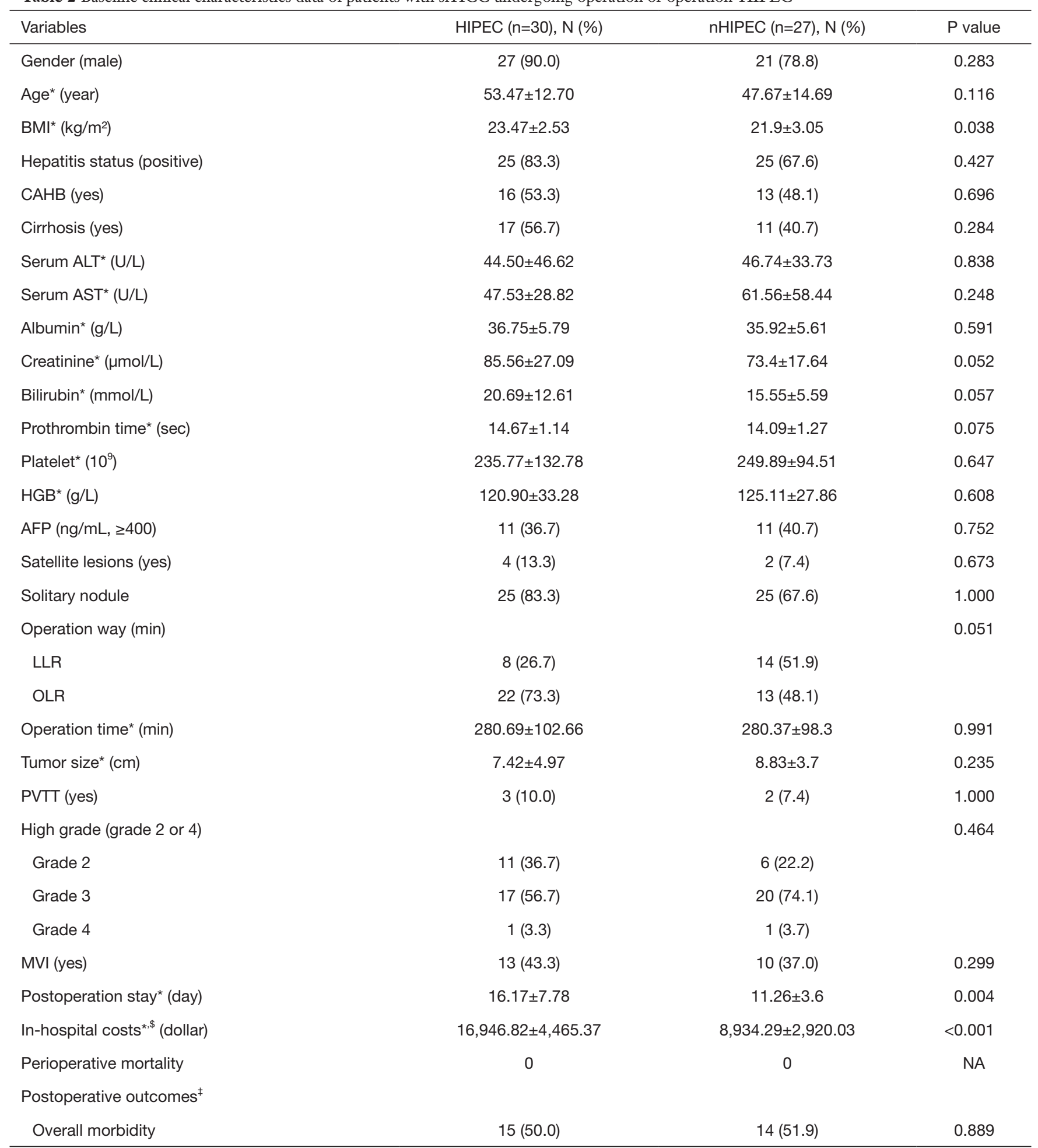

Table 2 (continued) 
Table 2 (continued)

\begin{tabular}{|c|c|c|c|}
\hline Variables & HIPEC (n=30), N (\%) & nHIPEC (n=27), N (\%) & $P$ value \\
\hline I-II & $14(46.7)$ & $12(44.4)$ & \\
\hline III-IV & $1(3.3)$ & $2(7.4)$ & \\
\hline V (death) & 0 & 0 & \\
\hline Bile leakage & 0 & 0 & 1.000 \\
\hline Peritoneal infection & $5(16.7)$ & $5(18.5)$ & 1.000 \\
\hline Hepatic dysfunction & $13(43.3)$ & $8(29.6)$ & 0.284 \\
\hline Pulmonary complications & $8(26.7)$ & $5(18.5)$ & 0.464 \\
\hline \multicolumn{4}{|l|}{ Recurrence } \\
\hline Intrahepatic & $13(43.3)$ & $14(51.9)$ & 0.696 \\
\hline Peritoneal dissemination & $3(10.0)$ & $3(11.1)$ & 0.892 \\
\hline Lung & $2(6.7)$ & $1(3.7)$ & 0.617 \\
\hline Bone & 0 & 0 & NA \\
\hline Brain & 0 & 0 & NA \\
\hline
\end{tabular}

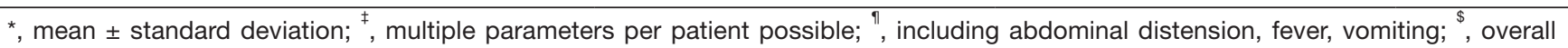
hospital costs for the inpatient episode were calculated initially in the Chinese yuan renminbi and converted to dollars at an exchange rate of 1 to 6.9298. srHCC, spontaneously ruptured hepatocellular carcinoma; HIPEC, hyperthermic intraperitoneal chemotherapy; BMI, body mass index; CAHB, chronic active hepatitis B; HGB, hemoglobin; AFP, alpha-fetoprotein; LLR, laparoscopic liver resection; OLR, open liver resection; PVTT, portal vein, tumor thrombus; MVI, microvascular invasion.

A

$\mp$ nHIPEC + HIPEC

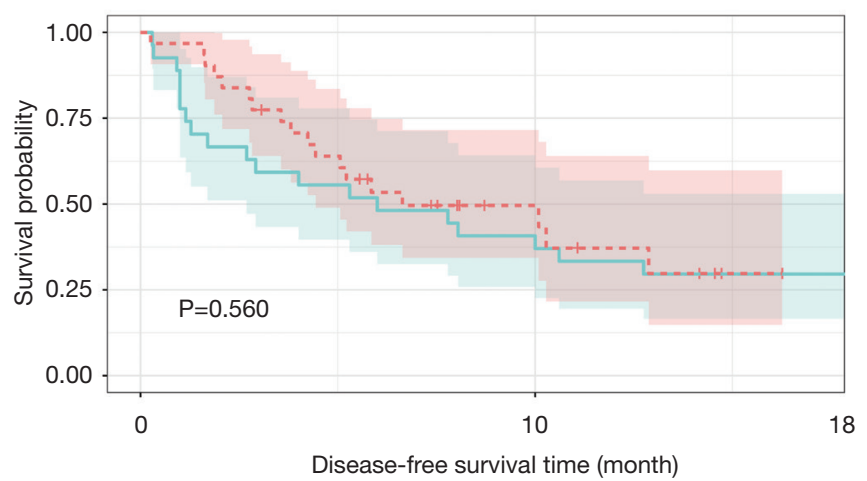

B

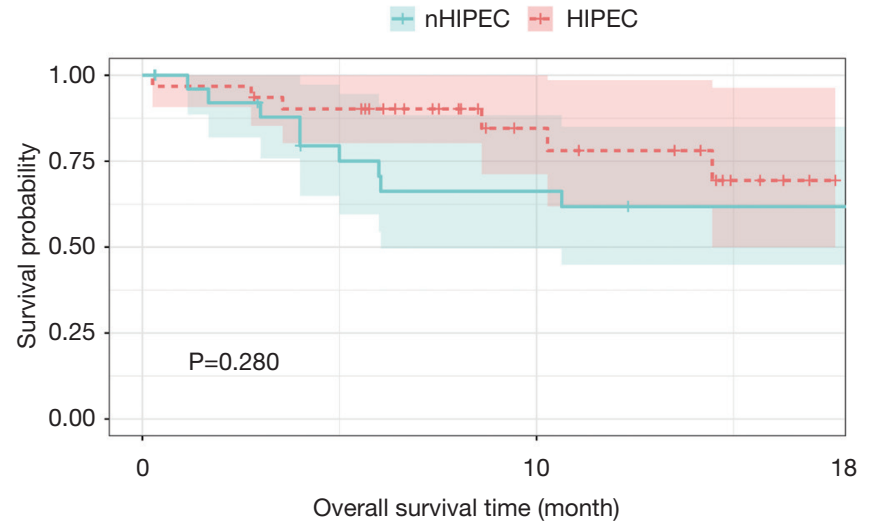

Figure 3 Comparison of survival rates between the HIPEC and nHIPEC groups (A,B). HIPEC, hyperthermic intraperitoneal chemotherapy. 
The pattern of recurrence included lung, peritoneal cavity, bone, and brain metastasis. The incidence of intraperitoneal implant metastasis was not significantly different between the HIPEC and the non-HIPEC patient $(\mathrm{P}=0.892)$. The intrahepatic recurrence rate in the HIPEC patient and the non-HIPEC patient was $43.3 \%$ and $51.9 \%$, respectively $(\mathrm{P}=0.696)$.

Univariate analysis showed that CAHB, ALT, AST, AFP, satellite lesions, number of tumors, and tumor size were associated with recurrence. Sex, AFP, and tumor size were correlated with OS. Multivariate Cox regression analyses did not find better clinical outcomes for patients treated with HIPEC, and HIPEC cannot be served as an independent predictor of clinical prognosis (Table 3).

\section{Discussion}

With the advance in operative techniques, the apparatus for hepatectomy, and perioperative management, surgical therapy has been proved to be a safe and effective method to treat HCC and has achieved encouraging survival prognosis. Tumor rupture is still a formidable clinical challenge for surgeons. The prognosis of patients with srHCC is abysmal, and the median survival time is 7 weeks to 11 months $(38,39)$. A Japanese study showed the 1-, 3 -, and 5-year survival rates for all patients undergoing hepatectomy for liver cancer were $87.8 \%, 69.2 \%$, and $53.4 \%$, respectively (40). Rupture of HCC means a later tumor stage, reflected by the tumor size, number of tumors, portal vein tumor thrombus, and microvascular invasion. A previous study showed that tumors on or protruding from the liver surface are more prone to rupture (41), but the present studies cannot test this issue, as detailed clinical data concerning the location and shape of the ruptured tumors was not obtained.

An earlier report showed that long-term survival could be expected if the patient can tolerate the liver resection of the ruptured tumor in selected cases. However, the recurrence rate of survival patients is as high as $67 \%$ to $100 \%$, with extrahepatic recurrence occurring in about half of these patients (39,42-44). A ruptured HCC is regarded as a T4 tumor, in the light of the TNM staging system, and is correlated with poor clinical outcomes, with tumor cell implanting in the abdominal cavity increasing the recurrence rate of cancer $(45,46)$. Distilled water lavages during surgery have been accepted as an established technique to prevent tumor cells disseminating after liver resection and have been proved to yield positive outcomes
$(47,48)$. However, this approach has limitations (3-5).

HIPEC has been widely applied after surgery for various abdominal malignant tumors with proven safety and feasibility. Since Spratt and others first reported the treatment mode of HIPEC in 1980, it has gradually become a mature treatment mode through continuous improvement by clinicians and scholars (8). However, in the present study in patients with srHCC, the comparison between the HPIEC group and the non-HIPEC group could not prove significant differences in OS and DFS. There is no sufficient evidence that intraperitoneal hyperthermic perfusion chemotherapy leads to added survival benefits for patients with srHCC, and HIPEC did not present a lower risk of abdominal metastasis. Although the incidence of postoperative complications has no statistical difference between the HIPEC group and the non-HIPEC group, additional treatment may reduce the quality of life of patients, and some patients experienced increased abdominal distension (32.3\%), fever (29.0\%), vomiting (19.3\%) and other additional complications after HIPEC. HIPEC increases the financial burden of patients and the length of hospitalization after the operation. There are still many problems with HIPEC treatment itself, including how to select the patients, a drug used in the treatment and specific operation (treatment, temperature, and time), and the corresponding complications and risk of death caused by the treatment (49).

There are still some questions about how HIPEC works inside the body. The mechanisms considered are: first, a nuclear mechanism mediates hyperthermia that inhibits DNA replication, transcription, and repair. After an hour of $43{ }^{\circ} \mathrm{C}$ hyperthermia, the tumor cells can be irreversibly killed (50). Second, under elevated temperature, the absorption rate and activity of chemotherapeutic drugs in the abdominal cavity is enhanced, and the anticancer effect is improved (51). Then, mechanical irrigation and the chemotherapy drugs act directly on free cancer cells (FCCs), which may be planted in the peritoneum and form nodules, in the abdominal cavity. Through intraperitoneal washing and direct action of chemotherapy drugs, HIPEC could reduce the risk of recurrence caused by FCCs (51). The HIPEC treatment in our study was lobaplatin or 5 -fluorouracil, and the treatment time was 60 minutes. Whether specific changes to other drugs and in treatment time and improvements to HIPEC operation will continue to improve the survival of these patients still needs to be further explored. Getting robust clinical data on whether different genotypes, primary tumor sites, and other factors 


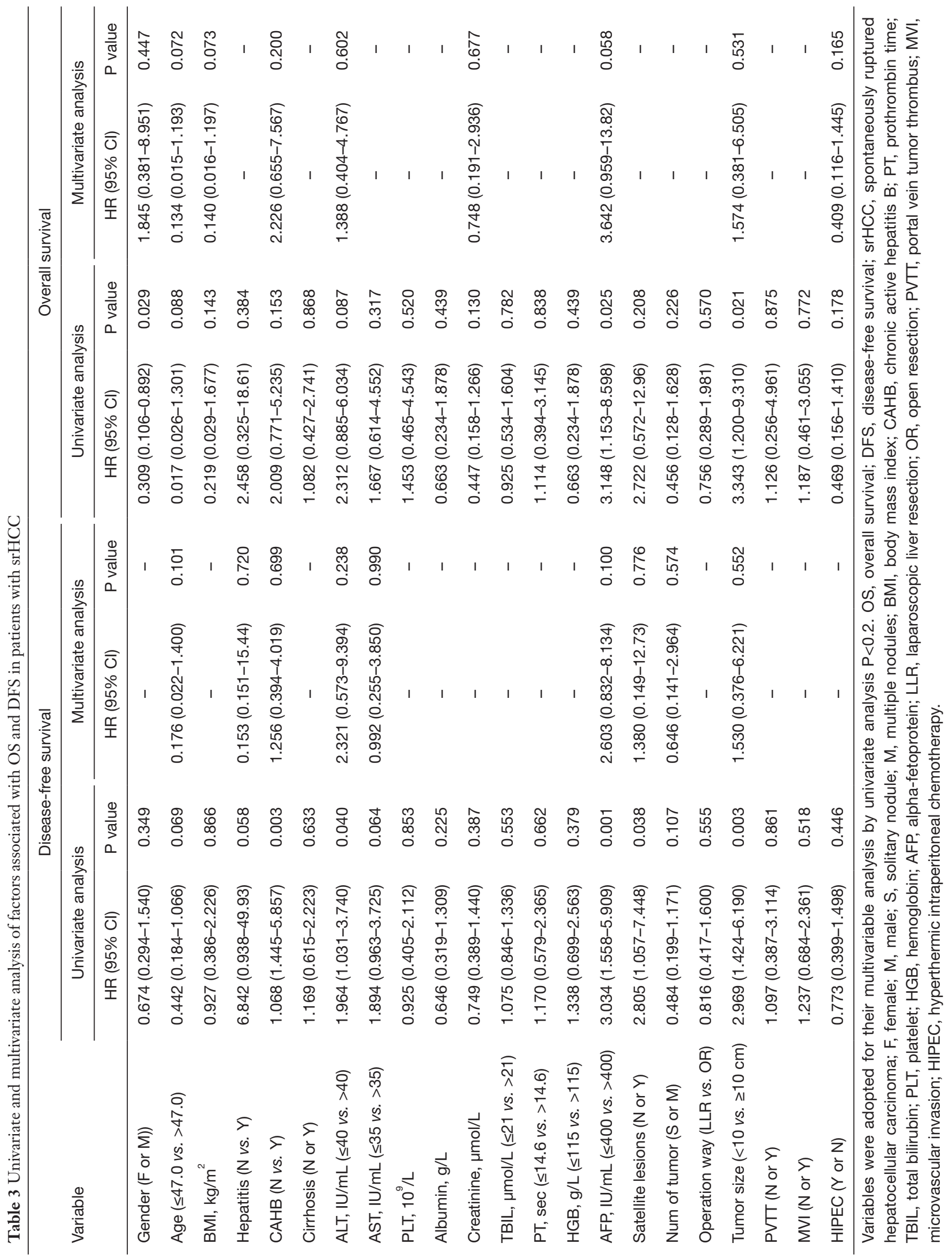


impact the therapeutic efficacy, we will provide a precise and effective treatment plan for patients with the peritoneal spread.

Finally, several limitations of our study should be mentioned. First, this is a retrospective study, and potential confounders and biases could not be avoided. A RCT can better reduce the selection biases in observational studies. However, considering that srHCC is a relatively rare event, it is critical to perform an RCT here; also, the setting of srHCC means patients usually need urgent treatment, and the process of identification, recruitment, and randomization may lead to clinically unacceptable treatment delays. Second, the occurrence of srHCC is rare, and this is a single-center study, so the sample size of this study is small, which may cause type 2 errors. In order to eliminate selection bias, PSM was performed in this research. Processed with the combination of clinical and pathological covariates, the propensity score matching made a comparable distribution of the clinicopathological characteristics between the cohorts, thus bringing about a result that was similar to random allocation. Third, given some cases, might be clinically silent because of how much rupture and bleeding, it is challenging to explain the complete spectrum of srHCC fully.

\section{Conclusions}

The prognosis of ruptured HCC patients was reduced compared with those of non-ruptured HCC patients. The addition of HIPEC after surgery did not influence the OS of DFS; there were more postoperative hospitalizations, more hospital expenses, and even more postoperative complications with HIPEC. The curative management of srHCC by surgery alone leads to a better quality of life. These results suggest HIPEC may not be a robust treatment for srHCC. More prospective and randomized data are needed to determine whether some selected patients with srHCC still receive help from HIPEC.

\section{Acknowledgments}

Funding: This study was supported by the Guangdong Medical Science and Technology Research Fund (A2018128), Natural Science Foundation of Guangdong Province (2017A030313548), the Startup Fund of National Natural Science Foundation of China (8197112215) and the Startup Fund of National Natural Science Foundation of China (8190103124).

\section{Footnote}

Reporting Checklist: The authors have completed the STROBE reporting checklist. Available at http://dx.doi. org/10.21037/atm-20-5829

Data Sharing Statement: Available at http://dx.doi. org/10.21037/atm-20-5829

Conflicts of Interest: All authors have completed the ICMJE uniform disclosure form (available at http://dx.doi. org/10.21037/atm-20-5829). The authors have no conflicts of interest to declare.

Etbical Statement: The authors are accountable for all aspects of the work in ensuring that questions related to the accuracy or integrity of any part of the work are appropriately investigated and resolved. The study was conducted in accordance with the Declaration of Helsinki (as revised in 2013). Institutional review board approval of our hospital was obtained for this study. This was a retrospective clinical study, which only analyzed the earlier clinical data. The data processed did not reveal the patient's identity information, so there was no need for ethical recognition and informed consent.

Open Access Statement: This is an Open Access article distributed in accordance with the Creative Commons Attribution-NonCommercial-NoDerivs 4.0 International License (CC BY-NC-ND 4.0), which permits the noncommercial replication and distribution of the article with the strict proviso that no changes or edits are made and the original work is properly cited (including links to both the formal publication through the relevant DOI and the license). See: https://creativecommons.org/licenses/by-nc$\mathrm{nd} / 4.0 \%$.

\section{References}

1. Torre LA, Bray F, Siegel RL, et al. Global cancer statistics, 2012. CA Cancer J Clin 2015;65:87-108.

2. Aoki T, Kokudo N, Matsuyama Y, et al. Prognostic impact of spontaneous tumor rupture in patients with hepatocellular carcinoma: an analysis of 1160 cases from a nationwide survey. Ann Surg 2014;259:532-42.

3. Huguet EL, Keeling NJ. Distilled water peritoneal lavage after colorectal cancer surgery. Dis Colon Rectum 2004;47:2114-9. 
4. Iitaka D, Shiozaki A, Ichikawa D, et al. Blockade of chloride ion transport enhances the cytocidal effect of hypotonic solution in gastric cancer cells. J Surg Res 2012;176:524-34.

5. Basha G, Ghirardi M, Geboes K, et al. Limitations of peritoneal lavage with antiseptics in prevention of recurrent colorectal cancer caused by tumor-cell seeding: experimental study in rats. Dis Colon Rectum 2000;43:1713-8.

6. Ohno S, Siddik ZH, Kido Y, et al. Thermal enhancement of drug uptake and DNA adducts as a possible mechanism for the effect of sequencing hyperthermia on cisplatininduced cytotoxicity in L1210 cells. Cancer Chemother Pharmacol 1994;34:302-6.

7. Panteix G, Beaujard A, Garbit F, et al. Population pharmacokinetics of cisplatin in patients with advanced ovarian cancer during intraperitoneal hyperthermia chemotherapy. Anticancer Res 2002;22:1329-36.

8. Spratt JS, Adcock RA, Muskovin M, et al. Clinical delivery system for intraperitoneal hyperthermic chemotherapy. Cancer Res 1980;40:256-60.

9. van de Vaart PJ, van der Vange N, Zoetmulder FA, et al. Intraperitoneal cisplatin with regional hyperthermia in advanced ovarian cancer: pharmacokinetics and cisplatinDNA adduct formation in patients and ovarian cancer cell lines. Eur J Cancer 1998;34:148-54.

10. van Driel WJ, Koole SN, Sonke GS. Hyperthermic Intraperitoneal Chemotherapy in Ovarian Cancer. N Engl J Med 2018;378:1363-4.

11. Vanounou T, Garfinkle R. Evaluation of Cytoreductive Surgery and Hyperthermic Intraperitoneal Chemotherapy for Peritoneal Carcinomatosis of Colorectal Origin in the Era of Value-Based Medicine. Ann Surg Oncol 2016;23:2556-61.

12. Yang SY, Kang JH, Kim HS, et al. Status of cytoreductive surgery and hyperthermic intraperitoneal chemotherapy in patients with peritoneal carcinomatosis from colorectal cancer. J Gastrointest Oncol 2019;10:1251-65.

13. Spiliotis J, Nikolaou G, Kopanakis N, et al. Hepatocellular Carcinoma Peritoneal Metastasis: Role of Cytoreductive Surgery and Hyperthermic Intraperitoneal Chemotherapy (HIPEC). Gulf J Oncolog 2017;1:20-3.

14. Mehta S, Schwarz L, Spiliotis J, et al. Is there an oncological interest in the combination of CRS/ HIPEC for peritoneal carcinomatosis of HCC? Results of a multicenter international study. Eur J Surg Oncol 2018;44:1786-92.

15. Hung KC, Yang KL, Huang GC, et al. Cytoreduction surgery and hyperthermic intraperitoneal chemotherapy for treating advanced peritoneal metastases of hepatocellular carcinoma. Pleura Peritoneum 2020;5:20190030.

16. Chen HC, Zhang JY. Clinical study of fluorouracil implants in prevention of peritoneal implantation after liver cancer rupture. Hepat Bili Pancreat Surg 2012;(24):316-8.

17. Schroeder RA, Marroquin CE, Bute BP, et al. Predictive indices of morbidity and mortality after liver resection. Ann Surg 2006;243:373-9.

18. Lim C, Dokmak S, Farges O, et al. Reoperation for posthepatectomy hemorrhage: increased risk of mortality. Langenbecks Arch Surg 2014;399:735-40.

19. Kepenekian V, Aloy MT, Magne N, et al. Impact of hyperthermic intraperitoneal chemotherapy on Hsp27 protein expression in serum of patients with peritoneal carcinomatosis. Cell Stress Chaperones 2013;18:623-30.

20. Jarnagin WR, Gonen M, Fong Y, et al. Improvement in perioperative outcome after hepatic resection: analysis of 1,803 consecutive cases over the past decade. Ann Surg 2002;236:397-406; discussion 406-7.

21. Fisher B, Gunduz N, Saffer EA. Influence of the interval between primary tumor removal and chemotherapy on kinetics and growth of metastases. Cancer Res 1983;43:1488-92.

22. Cho JY, Suh KS, Kwon CH, et al. Outcome of donors with a remnant liver volume of less than $35 \%$ after right hepatectomy. Liver Transpl 2006;12:201-6.

23. Belghiti J, Hiramatsu K, Benoist S, et al. Seven hundred forty-seven hepatectomies in the 1990s: an update to evaluate the actual risk of liver resection. J Am Coll Surg 2000;191:38-46.

24. Ellinger-Ziegelbauer H, Karasuyama H, Yamada E, et al. Ste20-like kinase (SLK), a regulatory kinase for polo-like kinase (Plk) during the $\mathrm{G} 2 / \mathrm{M}$ transition in somatic cells. Genes Cells 2000;5:491-8.

25. Ceelen WP, Hesse U, de Hemptinne B, et al. Hyperthermic intraperitoneal chemoperfusion in the treatment of locally advanced intra-abdominal cancer. Br J Surg 2000;87:1006-15.

26. Wu Q, Qin SK, Teng FM, et al. Lobaplatin arrests cell cycle progression in human hepatocellular carcinoma cells. J Hematol Oncol 2010;3:43.

27. Wang Y, Zheng WL, Ma WL. Lobaplatin inhibits the proliferation of hepatollular carcinoma through p53 apoptosis axis. Hepat Mon 2012;12:e6024.

28. Saris CP, van de Vaart PJ, Rietbroek RC, et al. In vitro 
formation of DNA adducts by cisplatin, lobaplatin and oxaliplatin in calf thymus DNA in solution and in cultured human cells. Carcinogenesis 1996;17:2763-9.

29. McKeage MJ. Lobaplatin: a new antitumour platinum drug. Expert Opin Investig Drugs 2001;10:119-28.

30. Harstrick A, Bokemeyer C, Scharnofkse M, et al. Preclinical activity of a new platinum analogue, lobaplatin, in cisplatin-sensitive and -resistant human testicular, ovarian, and gastric carcinoma cell lines. Cancer Chemother Pharmacol 1993;33:43-7.

31. Dindo D, Demartines N, Clavien PA. Classification of surgical complications: a new proposal with evaluation in a cohort of 6336 patients and results of a survey. Ann Surg 2004;240:205-13.

32. Rahbari NN, Garden OJ, Padbury R, et al. Posthepatectomy haemorrhage: a definition and grading by the International Study Group of Liver Surgery (ISGLS). HPB (Oxford) 2011;13:528-35.

33. Rahbari NN, Garden OJ, Padbury R, et al. Posthepatectomy liver failure: a definition and grading by the International Study Group of Liver Surgery (ISGLS). Surgery 2011;149:713-24.

34. Koch M, Garden OJ, Padbury R, et al. Bile leakage after hepatobiliary and pancreatic surgery: a definition and grading of severity by the International Study Group of Liver Surgery. Surgery 2011;149:680-8.

35. Dixit P, Dwivedi LK, Ram F. Estimating the impact of antenatal care visits on institutional delivery in India: A propensity score matching analysis. Health 2013;5:862-78.

36. Rubin DB. Using Propensity Scores to Help Design Observational Studies: Application to the Tobacco Litigation. Health Services and Outcomes Research Methodology 2001;2:169-88.

37. Ali Ahmed AH, Love TE, Gambassi G, et al. Heart failure, chronic diuretic use, and increase in mortality and hospitalization: an observational study using propensity score methods. Eur Heart J 2006;27:1431-9.

38. Zhu LX, Wang GS, Fan ST. Spontaneous rupture of hepatocellular carcinoma. Br J Surg 1996;83:602-7.

39. Liu CL, Fan ST, Lo CM, et al. Management of spontaneous rupture of hepatocellular carcinoma: singlecenter experience. J Clin Oncol 2001;19:3725-32.

40. Ikai I, Arii S, Okazaki M, et al. Report of the 17th Nationwide Follow-up Survey of Primary Liver Cancer in Japan. Hepatol Res 2007;37:676-91.

41. Chen CY, Lin XZ, Shin JS, et al. Spontaneous rupture of hepatocellular carcinoma. A review of 141 Taiwanese cases and comparison with nonrupture cases. J Clin Gastroenterol 1995;21:238-42.

42. Miyoshi A, Kitahara K, Kohya N, et al. Outcomes of patients with spontaneous rupture of hepatocellular carcinoma. Hepatogastroenterology 2011;58:99-102.

43. Chok KS, Cheung TT, Chan SC, et al. Surgical outcomes in hepatocellular carcinoma patients with portal vein tumor thrombosis. World J Surg 2014;38:490-6.

44. Oba A, Takahashi S, Kato Y, et al. Usefulness of resection for hepatocellular carcinoma with macroscopic bile duct tumor thrombus. Anticancer Res 2014;34:4367-72.

45. Sonoda T, Kanematsu T, Takenaka K, et al. Ruptured hepatocellular carcinoma evokes risk of implanted metastases. J Surg Oncol 1989;41:183-6.

46. Li Y, Zhou YF, Liang H, et al. Chinese expert consensus on cytoreductive surgery and hyperthermic intraperitoneal chemotherapy for peritoneal malignancies. World J Gastroenterol 2016;22:6906-16.

47. Chang YM, Hsu KF, Yu JC, et al. Distilled water peritoneal lavage in patients with rupture hepatocellular carcinoma. Hepatogastroenterology 2013;60:140-3.

48. Zhou SJ, Zhang EL, Liang BY, et al. Distilled Water Lavage During Surgery Improves Long-Term Outcomes of Patients with Ruptured Hepatocellular Carcinoma. J Gastrointest Surg 2015;19:1262-70.

49. Yan TD, Morris DL. Cytoreductive surgery and perioperative intraperitoneal chemotherapy for isolated colorectal peritoneal carcinomatosis: experimental therapy or standard of care? Ann Surg 2008;248:829-35.

50. Garofalo A, Valle M, Garcia J, et al. Laparoscopic intraperitoneal hyperthermic chemotherapy for palliation of debilitating malignant ascites. Eur J Surg Oncol 2006;32:682-5.

51. Kusamura S, Dominique E, Baratti D, et al. Drugs, carrier solutions and temperature in hyperthermic intraperitoneal chemotherapy. J Surg Oncol 2008;98:247-52.

(English Language Editor: J. Chapnick)

Cite this article as: Ruan S, Shi N, Chen Z, Han H, Wang H, Jin L, Zou Y, Zhang Y, Yu M, Jin H. The role of hyperthermic intraperitoneal chemotherapy in the treatment of spontaneously ruptured hepatocellular carcinoma: a pilot study. Ann Transl Med 2020;8(18):1132. doi: 10.21037/atm-20-5829 